\title{
Improving tumour heterogeneity MRI assessment with histograms
}

\author{
N Just ${ }^{\star}, 1$ \\ ${ }^{1}$ Centre d'Imagerie Biomédicale (CIBM) Animal Imaging and Technology Core, Ecole Polytechnique Fédérale de Lausanne, \\ Lausanne, Switzerland
}

By definition, tumours are heterogeneous. They are defined by marked differences in cells, microenvironmental factors (oxygenation levels, pH, VEGF, VPF and TGF- $\alpha$ ) metabolism, vasculature, structure and function that in turn translate into heterogeneous drug delivery and therapeutic outcome. Ways to estimate quantitatively tumour heterogeneity can improve drug discovery, treatment planning and therapeutic responses. It is therefore of paramount importance to have reliable and reproducible biomarkers of cancerous lesions' heterogeneity. During the past decade, the number of studies using histogram approaches increased drastically with various magnetic resonance imaging (MRI) techniques (DCE-MRI, DWI, SWI etc.) although information on tumour heterogeneity remains poorly exploited. This fact can be attributed to a poor knowledge of the available metrics and of their specific meaning as well as to the lack of literature references to standardised histogram methods with which surrogate markers of heterogeneity can be compared. This review highlights the current knowledge and critical advances needed to investigate and quantify tumour heterogeneity. The key role of imaging techniques and in particular the key role of MRI for an accurate investigation of tumour heterogeneity is reviewed with a particular emphasis on histogram approaches and derived methods.

\section{TUMOUR HETEROGENEITY: METHODS ARE NEEDED FOR AN APPROPRIATE CHARACTERISATION}

Investigating tumour heterogeneity is a key point in cancer research because inter- and intra-tumour differences have so far limited the development of targeted therapies for patients. Heterogeneity in tumours already exists at the cell level and is highly influenced by this cell's genetic background and origin as well as the environment where it establishes (De Sousa et al, 2013). The wide varieties of genetic, cellular and molecular mutations that may occur during the course of tumour development or as a response to treatment are complex. Their characterisation becomes even more difficult if these mutations occur in individual cancers or in tumour subtypes. This has direct implications on the clinical outcome as well as the development of adequate therapies. Intratumour heterogeneity prevents an adequate clinical diagnosis and is involved in tumour resistance. The identified existence of multiple clones within a tumour orientates newer therapies towards more personalised treatments including those targeting specific clone subsets. Although appropriate indices of heterogeneity have already shown to be good predictors of cancer progression, ways to investigate and appreciate the extent of this heterogeneity are still lacking due in part to a poor understanding of the molecular mechanisms underlying it.

A better and more systematic appreciation of intra and intertumour heterogeneity is crucial for drug development as well as for the accurate assessment of response to treatment. In addition, although combination therapies have proved their value, a better understanding and quantification of tumour heterogeneity should improve the design of therapeutic schemes (where and when a drug must be introduced).

Finally, the speed of development of therapeutic agents will also be increased, whereas costs will be drastically limited. These improvements may be explained by the faster determination of the different vascular and cellular components of a tumour based on improved characterisation of heterogeneity and guided therapeutic schemes. Targeted approaches such as personalised treatment aim to produce greater effectiveness and less toxicity. Efforts are under

*Correspondence: Dr N Just; E-mail: nathalie.just@epfl.ch

Received 31 March 2014; revised 4 August 2014; accepted 6 August 2014;

published online 30 September 2014

(c) 2014 Cancer Research UK. All rights reserved 0007-0920/14

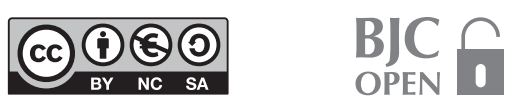


way to target responder populations (e.g., aromatase inhibitors for women that slowly metabolise tamoxifen and that may be at increased risk of cancer progression) rather than assuming that all the patients can receive treatments to the same extent. Thus, a better characterisation of cancer biology with non-invasive methods in conjunction with an enhanced knowledge of the molecular, metabolic and genomic profile of tumours and their subsets helps the understanding of the tumour environment and the development of new clinically relevant biomarkers. These can identify drug sensitivity for a faster enrolment of candidates who will benefit from a treatment under study. As a consequence, the cost-effectiveness of targeted drugs should be demonstrated. Moreover, faster decisions will be taken on whether to continue or stop drug development avoiding at the same time further exposure of experimental animals and patients and further costs.

The evaluation of tumour heterogeneity improved tremendously with the advent of imaging techniques such as optical imaging techniques, positron emission tomography and magnetic resonance imaging (MRI) both at the clinical and experimental levels. The combination of these imaging modalities can provide greater insights into characterisation of tumour heterogeneity: cancers display various kinds of heterogeneity such as cellular morphology, gene expression, metabolism, angiogenic and proliferative potential that can be specifically investigated using optical imaging or positron emission tomography for molecular and metabolic imaging and MRI for investigating angiogenic processes (DCEMRI) and cellular morphology (diffusion). Here, we focus on MRI techniques.

The strength of MRI compared with other imaging modalities (CT, positron emission tomography) resides in its potential to provide a vast array of different image contrasts (T1, T2, diffusion-weighted, flow-weighted, magnetisation transfer, chemical exchange saturation transfer, etc.) at a high spatial resolution, in a three dimensional manner and non-invasively providing unique insight into tumour heterogeneity. Various physiological parameters have been estimated on a voxel by voxel basis allowing a full visualisation of the tumour heterogeneity during its progression or during its regression following a treatment. However, in many studies, average parameters over entire regions of interest are calculated and compared. The methodologies for calculating and interpreting voxel by voxel values are poorly known and may be time-consuming. Unfortunately, mean and median quantitative values are not always significantly sensitive to small changes or treatment effects. In addition, they may not represent the precise status of the tumour owing to the intrinsic chaotic environment of tumours, which may be drastically different between tumours of the same type or within tumours where one part may be viable and the other not. With increasing advances in both high-resolution MRI and signal processing methods, histogram analysis of cancer MRI is more and more used. This methodology showed its usefulness for investigating the distributions of various tumour parameters such as permeability in dynamic contrast-enhanced MRI (DCE-MRI) (Hayes et al, 2002; Padhani, 2002; Peng et al, 2012), vessel size index and blood volumes (VSI, CBV) (Just, 2011; Burrell et al, 2012) and apparent diffusion coefficient (ADC) in diffusion MRI (Downey et al, 2013).

Principles of first-order histogram analysis, parameters. The histogram of an image is a function showing (for each intensity level) the number of pixels in the whole image having the same intensity. It describes in a simple manner the statistical information contained in the image. Most histogram analyses use descriptive parameters to characterise and compare distributions of tumour biomarkers in a quantitative manner. A descriptive analysis reports the following quantitative factors: mean, standard deviation, mode, maximum and minimum, kurtosis, skewness, and percentiles as well as entropy. These parameters represent the first-order statistical properties of the image. Mean and standard deviations represent average and dispersion of the histogram, respectively. The mode represents the value with highest counts. Kurtosis, skewness and entropy are usually more difficult to apprehend and interpret. Kurtosis reflects the peakedness of the distribution and is a measure of the shape of the probability distribution (Figure 1). Skewness represents a measure of asymmetry of the probability distribution. The entropy represents a statistical measure of the 'irregularities' in a histogram. Entropy is a recognised parameter allowing describing the variation of a parameter of interest's distribution. Finally, a percentile represents the value below which a percentage of observations is calculated. Apart from these statistical and mathematical considerations, the meaning of these metrics in cancer studies is ambiguous (Figure 1). Although many studies agree that histogram analyses add information for discriminating between benign and malignant regions (Carter
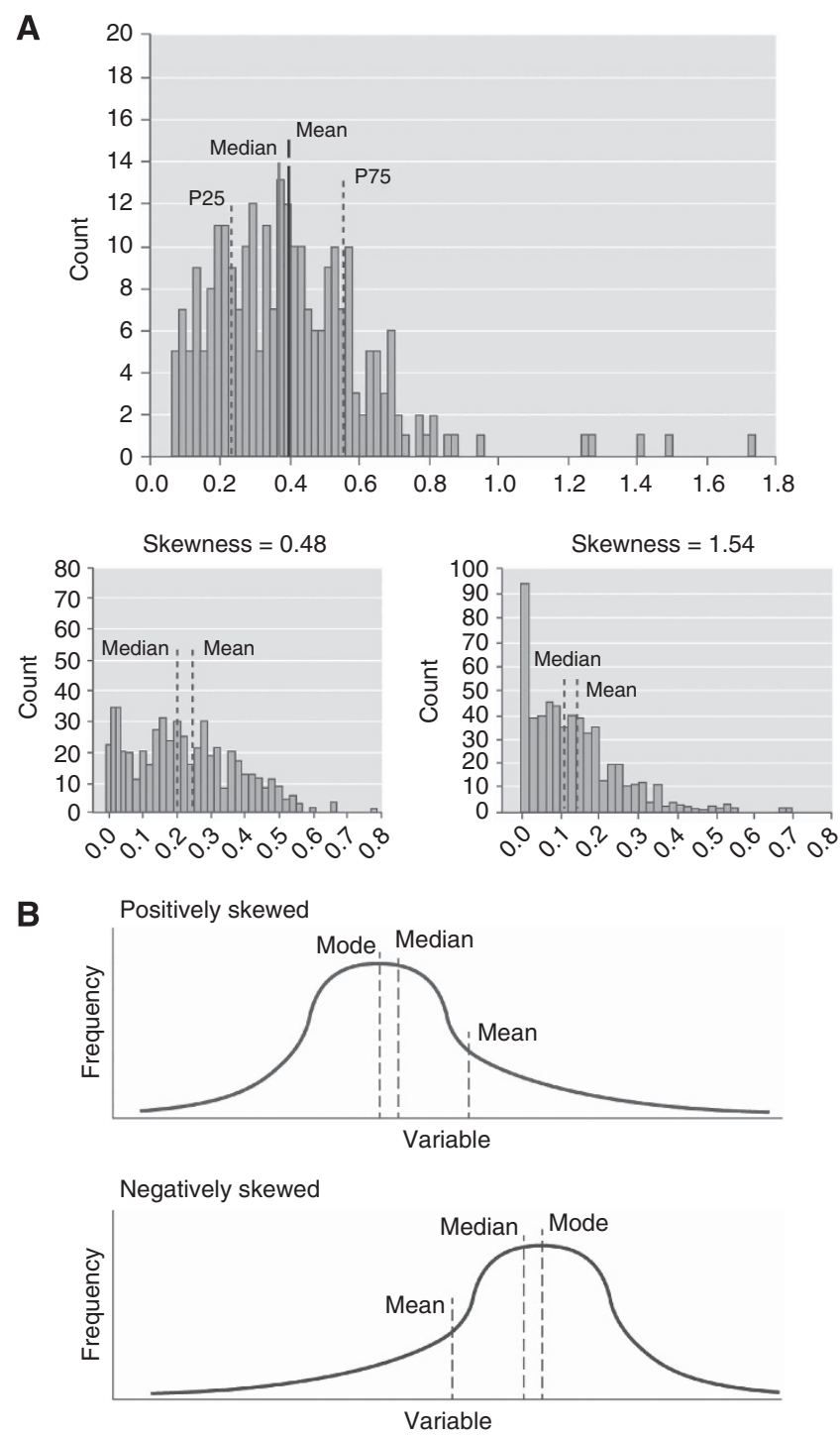

Figure 1. Interpretation of the properties of histograms. (A). Definition of mean, median and percentiles. P2 $5=25$ th percentile; P75 $=75$ th percentile. (B). Kurtosis (K) Platykurtosis indicates a flatter peak with negative kurtosis (left, $K<0$ ), and leptokurtosis indicates a sharp peak with positive kurtosis (Right, $K>0$ ). Skewness: if a histogram has an elongated tail on the left side of the mean, it is negatively skewed. If a histogram has an elongated tail on the right side of the mean, it is positively skewed. 
et al, 2013) or for a better characterisation of response to treatment, very few of them try to interpret the true meaning of kurtosis, skewness and percentile parameters.

Until recently, a majority of clinical studies using histograms were performed in the brain (see Table 1) less contaminated by motion. Nevertheless, histograms are more and more used in extracranial areas such as endometrial cancer (Woo et al, 2013), renal cell cancer (Chandarana et al, 2012), and cervical and breast cancer (Johansen et al, 2009; Yuh et al, 2009). In Table 1, a list of recent studies is provided, showing that histogram factors can be significant predictors of response to treatment in various types of clinical and experimental cancers (King et al, 2013). Mean, median and percentiles are the histogram parameters mostly investigated although skewness and kurtosis were evaluated more often recently (Baek et al, 2012; Chandarana et al, 2012; Downey et al, 2013; King et al, 2013). Entropy has not been widely used to date but the increased investigation of cancers with texture analysis, which can be seen as a second order histogram analysis methodology, should certainly change this (Foroutan et al, 2013). The majority of studies have investigated distributions of ADC, CBV and DCE-MRI parameters such as $\mathrm{K}^{\text {trans }}$ or extravascular extracellular space (EES) although various other DCE-MRI characteristics such as lesion enhancement, wash in slope or area under the curve have been investigated with histograms (Table 1).

Histogram analysis has mostly been performed on data collected from manually drawn regions of interest (ROI) (Baek et al, 2012; Foroutan et al, 2013; King et al, 2013; Song et al, 2013; Woo et al, 2013). Although ROIs were drawn by trained observers and/or radiologists in clinical studies, ROI-based methods include interobserver variability in ROI positioning and misregistration between pre- and post-contrast-enhanced images that can create confounds in histograms. As an alternative, Chandarana et al (2012) proposed the use of a segmentation algorithm on preregistered pre- and post-contrast images of renal cell cancer (RCC) subtypes. The assessment of whole lesion enhancement descriptive histogram parameters using semi-automated programs showed significant increases in sensitivity, specificity and accuracy in the diagnosis of RCC compared with ROI analysis.

Kurtosis, skewness and percentiles as biomarkers of tumour heterogeneity. Kurtosis, skewness and percentiles appear to be promising parameters for differentiating between different types of gliomas (Just, 2011), between pseudoprogression and early tumour progression in glioblastomas (Baek et al, 2012; Song et al, 2013) and between cancer subtypes (Chandarana et al, 2012). Percentiles, kurtosis and skewness of ADC distributions were correlated to histological grade with statistical tests and allowed grading of endometrial cancer (Woo et al, 2013) and differentiation between cervical cancers (Downey et al, 2013). Baek et al (2012) examined the predictive value of percentiles, skewness and kurtosis for discrimination between pseudoprogression and progression of glioblastomas. Patients with newly diagnosed glioblastomas that developed enlarged contrast-enhanced lesions after chemotherapy and radiation therapy following surgical resection underwent perfusion MRI. A classification of the percent change in skewness and kurtosis of the normalised cerebral blood volume (nCBV) during the early treatment period was performed allowing the characterisation of predictors of tumour regression by means of a multivariable logistic regression analysis. Patients were categorised into two groups: those with early tumour progression and those with pseudoprogression both confirmed by pathologic analysis. Visual analysis of histographic patterns of nCBV histograms demonstrated that post- chemotherapy and radiation therapy contrast-enhanced lesions classified as pseudoprogression had positive changes in kurtosis and skewness and lower range, mode and maximum than lesions classified as early tumour progression. In pseudoprogression, lesions demonstrated positive changes in kurtosis and skewness as well as leptokurtosis whereas negative changes in kurtosis and skewness and platykurtosis were found in early tumour progression. Furthermore, regression and receiver operating characteristic analyses demonstrated significant associations of histographic patterns with early tumour progression. Song et al (2013) completed this study using the same methodology to differentiate true progression from pseudoprogression using histogram analysis of ADC. The 5th percentile of the cumulative histogram analysis was the only independently differentiating variable in the multivariate analysis.

Histogram parameters (mean, median, 75th percentile) were significantly higher in clear cell RCC (ccRCC) than in the papillary subtype of RCC (pRCC) (Chandarana et al, 2012). However, kurtosis and skewness were significantly lower in ccRCC. In addition, an accurate choice of cutoff values for histogramdescriptive parameters drastically increased the accuracy, specificity and sensitivity of the discrimination of ccRCC from p. Finally, kurtosis had the best interreader agreement in discriminating ccRCC from pRCC. As mentioned by the authors, their study was limited by 'a priori' knowledge of lesion classification and by the needs to extend to other less occurring RCC subtypes to demonstrate its true value.

Histogram approaches also showed their added value as predictors of response to treatment in various clinical and preclinical cancers (Chang et al, 2004; Johansen et al, 2009; Peng et al, 2012; Shukla-Dave et al, 2012; Foroutan et al, 2013; King et al, 2013; Mignion et al, 2014). Skewness and kurtosis of ADC and $\mathrm{K}^{\text {trans }}$ distributions were used as predictors of treatment response in head and neck squamous cell carcinoma (HNSCC) (ShuklaDave et al, 2012; King et al, 2013). King et al (2013) examined the value of diffusion-weighted imaging (DWI) for the prediction of treatment failure in primary stage III and IV HNSCC. Histogram analysis of ADC in pre- and post-radiation therapy was able to predict local failure within 2 years of treatment. Strong and significant trends for higher mean $\mathrm{ADC}$ and $\mathrm{ADC}$ skewness and kurtosis were identified in HNSCC tumours that failed treatment. Shukla-Dave et al (2012), on the other hand, wanted to assess the potential of DCE-MRI as a prognostic value of pre-treatment (surgery or chemoradiation therapy) in HNSCC patients and found that skewness of $\mathrm{K}^{\text {trans }}$ was a strong predictor of progression-free survival and overall survival in HNSCC patients with stage IV nodal disease. Histogram approaches and in particular, skewness and kurtosis parameters demonstrated their added value when investigating the same cancer type with different MRI techniques. Histogram analysis of HNSCC lesions allowed prediction of patient outcome pre- and post treatment using DCEMRI and DWI, respectively. Changes in the asymmetry of ADC and $\mathrm{K}^{\text {trans }}$ distributions may reveal different structural and functional characteristics of HNSCC lesions before and after treatment. These tumours are generally characterised by heterogeneous regions of hypoxia and necrosis contributing to poor treatment response because of resistance to radiation therapy. Hypoxic regions are generally less perfused within tumours and have shown increased asymmetric distributions (skewness) of transfer constants $\left(\mathrm{K}^{\text {trans }}, \mathrm{K}_{\mathrm{ep}}\right)$ in DCE-MRI, indicating poor perfusion. Tumour necrotic areas may have higher ADC values than in non-necrotic areas. Therefore, high ADC may be predictive of a poor treatment response. In primary HNSCC lesions, higher $\mathrm{ADC}$ skewness and kurtosis and a small increase of the percentage change of mean ADC showed local failure of an early radiation therapy treatment indicative of intratumoural low oxygen tensions. Histogram analysis of HNSCC not only provided predictors of patient outcome but also was able to inform regarding the tumour environment. This may be useful to identify patients requiring a transition to a more aggressive treatment or combination of treatments or even surgery. In both studies of HNSCC, statistical analysis of histogram parameters was performed using univariate 
Table 1. Fundamental and clinical studies using histogram analysis

\begin{tabular}{|c|c|c|c|c|c|c|}
\hline Study & $\begin{array}{l}\text { Clinical/ } \\
\text { experimental }\end{array}$ & Cancer & Parameter & Histogram analysis & $P$-value & Features \\
\hline Baek et al, 2012 & Human & Glioblastoma & CBV & $\begin{array}{l}\text { Skewness } \\
\text { Kurtosis } \\
\text { Histographic pattern } \\
\text { Range } \\
\text { Mode }\end{array}$ & $\begin{array}{l}0.02 \\
0.035 \\
0.002 \\
0.002 \\
0.005\end{array}$ & $\begin{array}{l}\text { Testing the predictive value of skewness } \\
\text { and kurtosis } \\
\text { Differentiating early tumour progression } \\
\text { from pseudoprogression }\end{array}$ \\
\hline Song et al, 2013 & Human & Glioblastoma & $\begin{array}{l}\text { ADC } \\
\text { CBV }\end{array}$ & $\begin{array}{l}\text { 5th percentile } \\
\text { 95th percentile }\end{array}$ & $\begin{array}{l}0.009 \\
0.015\end{array}$ & $\begin{array}{l}\text { True progression vs pseudoprogression } \\
\text { True progression vs pseudoprogression }\end{array}$ \\
\hline Just, 2011 & Rat & Gliomas & VSI, CBV & $\begin{array}{l}\text { 25th percentile, } 75 \text { th } \\
\text { percentile }\end{array}$ & $<0.023$ & VSI and BV of human and rodent gliomas \\
\hline Peng et al, 2012 & Human & Brain tumours & $\begin{array}{l}\text { Wash in } \\
\text { slope }\end{array}$ & $\mathrm{mFWHM}$ & $<0.001$ & $\begin{array}{l}\text { Evaluation of tumour brain response to } \\
\text { radiotherapy }\end{array}$ \\
\hline Tozer et al, 2007 & Human & $\begin{array}{l}\text { Low-grade gliomas: } \\
\text { oligodendroglioma, } \\
\text { oligoastrocytoma }\end{array}$ & $A D C$ & $\begin{array}{l}\mathrm{PH} ; \mathrm{PL} ; \text { mean value; } \\
\text { 10th, 25th, 50th, 75th } \\
\text { and 90th, skewness }\end{array}$ & & $\begin{array}{l}\text { To investigate whether low-grade } \\
\text { astrocytomas and oligodendrogliomas } \\
\text { exhibit different ADC histogram } \\
\text { characteristics because of their biological } \\
\text { difference and whether diagnoses of } \\
\text { tumour subtype can be suggested at } \\
\text { presentation using the histogram alone }\end{array}$ \\
\hline Kim et al, 2013 & Human & $\begin{array}{l}\text { Low-grade gliomas } \\
\text { vs high-grade } \\
\text { gliomas }\end{array}$ & CBV & $\begin{array}{l}\text { 99th percentile } \\
\text { Mean }\end{array}$ & $\begin{array}{r}<0.001 \\
0.014\end{array}$ & $\begin{array}{l}\text { To retrospectively assess the usefulness of a } \\
\text { cumulative CBV histogram for grading } \\
\text { gliomas }\end{array}$ \\
\hline Nowosielski et al, 2011 & Human & $\begin{array}{l}\text { Recurrent high- } \\
\text { grade glioma }\end{array}$ & ADC & Change in skewness & $P=0.04$ & $\begin{array}{l}\text { To evaluate ADC maps to distinguish anti- } \\
\text { vascular and anti-tumour effects in the } \\
\text { course of anti-angiogenic treatment of } \\
\text { rHGG }\end{array}$ \\
\hline $\begin{array}{l}\text { Rodriguez Gutierrez } \\
\text { et al, } 2014\end{array}$ & Human & $\begin{array}{l}\text { Paediatric posterior } \\
\text { fossa tumours }\end{array}$ & ADC & $\begin{array}{l}\text { Skewness, kurtosis mode } \\
10 \%, 25 \%, 50 \%, 75 \% \\
\text { and } 90 \% \text { energy, } \\
\text { entropy, mean variance, } \\
\text { texture analysis and } \\
\text { parameters }\end{array}$ & & $\begin{array}{l}\text { Improving current posterior fossa } \\
\text { discrimination of histological tumour type }\end{array}$ \\
\hline Chandarana et al, 2012 & Human & Renal cell cancer & $\begin{array}{l}\text { Lesion } \\
\text { enhancement }\end{array}$ & $\begin{array}{l}\text { Mean } \\
\text { Median } \\
\text { 75th percentile } \\
\text { Kurtosis } \\
\text { Skewness }\end{array}$ & $\begin{array}{l}<0.001 \\
<0.001 \\
<0.001 \\
<0.001 \\
<0.001\end{array}$ & Discriminating ccRCC from $\mathrm{pRCC}$ \\
\hline Woo et al, 2013 & Human & Endometrial cancer & ADC & $\begin{array}{l}\text { 25th, 75th, 90th, 95th } \\
\text { between grades } \\
\text { 25th, 75th, 90th, 95th }\end{array}$ & $\begin{array}{l}<0.03 \\
<0.024\end{array}$ & $\begin{array}{l}\text { Histogram analysis of endometrial cancer } \\
\text { ADC provides potential parameters for } \\
\text { grading preoperative tumours }\end{array}$ \\
\hline Shukla-Dave et al, 2012 & Human & HNSCC & $\mathrm{K}^{\text {trans }}$ & Skewness & $<0.001$ & $\begin{array}{l}\text { Assessment of the value of DCE-MRI in } \\
\text { HNSCC patients with nodal disease } \\
\text { undergoing chemotherapy }\end{array}$ \\
\hline King et al, 2013 & Human & HNSCC & $A D C$ & $\begin{array}{l}\text { Mean } \\
\text { Kurtosis } \\
\text { Skewness }\end{array}$ & $\begin{array}{l}0.112 \\
0.04 \\
0.015\end{array}$ & Prediction of treatment failure in HNSCC \\
\hline Crokart et al, 2005 & Mice & $\begin{array}{l}\text { Liver tumours, } \\
\text { fibrosarcomas }\end{array}$ & $\begin{array}{l}\mathrm{K}^{\text {trans }}, \mathrm{Kep}, \\
\mathrm{vp}\end{array}$ & $\begin{array}{l}\text { Mean histographic } \\
\text { patterns }\end{array}$ & $<0.05$ & $\begin{array}{l}\text { Explore anti-inflammatory drugs for } \\
\text { radiosensitisation of tumours }\end{array}$ \\
\hline Johansen et al, 2009 & Human & $\begin{array}{l}\text { Advanced breast } \\
\text { cancer }\end{array}$ & $\begin{array}{l}\text { Relative SI, } \\
\text { AUC (DCE- } \\
\text { MRI) }\end{array}$ & $\begin{array}{l}\text { Mean and 10th } \\
\text { percentile }\end{array}$ & 0.02 & $\begin{array}{l}\text { Early prediction of response to neoadjuvant } \\
\text { therapy }\end{array}$ \\
\hline Kyriasi et al, 2011 & Human & $\begin{array}{l}\text { Metastatic ovarian } \\
\text { cancer } \\
\text { Primary peritoneal } \\
\text { cancer }\end{array}$ & ADC & $\begin{array}{l}\text { Mean, 10th, 25th, 50th, } \\
\text { 75th, 90th } \\
\text { Kurtosis, skewness }\end{array}$ & $\begin{array}{l}<0.001 \\
<0.006\end{array}$ & $\begin{array}{l}\text { Evaluation of ADC histograms for predicting } \\
\text { chemotherapy response. Significance was } \\
\text { reached after the third course }\end{array}$ \\
\hline
\end{tabular}




\begin{tabular}{|c|c|c|c|c|c|c|}
\hline Study & $\begin{array}{l}\text { Clinical/ } \\
\text { experimental }\end{array}$ & Cancer & Parameter & Histogram analysis & $P$-value & Features \\
\hline Chang et al, 2004 & Human & Breast cancer & $\begin{array}{l}\text { Amplitude } \\
\text { Kout } \\
\text { Peak } \\
\text { enhancement }\end{array}$ & $\begin{array}{l}\text { Mean, s.d., kurtosis, } \\
\text { skewness }\end{array}$ & $<0.05$ & $\begin{array}{l}\text { Angiogenic response to neoadjuvant } \\
\text { chemotherapy } \\
\text { Before, after first and after final } \\
\text { chemotherapy }\end{array}$ \\
\hline Yuh et al, 2009 & Human & Cervical cancer & $\begin{array}{l}\text { Tumour } \\
\text { volume } \\
\text { Signal } \\
\text { intensity }\end{array}$ & 2.5th to 20th percentile & $<0.008$ & $\begin{array}{l}\text { Predictive power of MRI perfusion } \\
\text { parameters } \\
\text { During early treatment }\end{array}$ \\
\hline Downey et al, 2013 & Human & Cervical cancer & ADC & $\begin{array}{l}\text { 10th, 25th, 50th, 70th, } \\
\text { 90th percentiles } \\
\text { Skewness, kurtosis }\end{array}$ & $\begin{array}{r}<0.05 \\
0.016\end{array}$ & $\begin{array}{l}\text { Differentiation of cervical tumours } \\
\text { according to their histological } \\
\text { characteristics }\end{array}$ \\
\hline Foroutan et al, 2013 & Mice & Sarcoma & ADC & $\begin{array}{l}\text { \% change in mean ADC } \\
\text { Skewness } \\
\text { Kurtosis } \\
\text { Entropy }\end{array}$ & $\begin{array}{c}<0.007 \\
(\mathrm{D} 2) \\
\\
0.018 \\
0.043 \\
>0.05 \\
0.023 \\
\text { (Gems } \\
\text { only or } \\
\text { MK1775 } \\
\text { only) }\end{array}$ & $\begin{array}{l}\text { Evaluation of the combination of } \\
\text { gemcitabine and MK1775 treatment at } \\
\text { early time points }\end{array}$ \\
\hline
\end{tabular}

as well as multivariate logistic regression analyses to assess the statistical significance of the combinations of prognostic variables (Shukla-Dave et al, 2012) or to predict outcome and time to recurrence (King et al, 2013). Almost all the clinical studies presented in this review used univariate and multivariate statistical analyses to statistically correlate the value of either DCE-MRI parameters or DWI parameters with survival, age or sex. Most of these studies also included receiver operating characteristic analyses allowing the identification of the optimal threshold of any significant histogram parameter as well as maximisation of accuracy, sensitivity and specificity. These statistical evaluations are usually absent from preclinical cancer studies (Just, 2011; Foroutan et al, 2013; Mignion et al, 2014), but should definitely be investigated in preclinical models where histogram parameters could be used to evaluate their translational value to the clinics. King et al (2013) stated that the appropriate definition of ADC threshold is of clinical interest and in HNSCC lesions, knowledge of the high positive skewness and kurtosis and their thresholds altogether were predictive of treatment failure.

Thus, changes in histogram shape and asymmetry reflect microstructural and functional differences in tumour composition that might be of relevant interest for the development of therapeutic strategies in cancer. These parameters could also represent a step forward to considering MRI as a non-invasive 'histological' method (Just, 2011; Friedman et al, 2012). In this regard, preclinical studies have the advantage of providing histological evaluation more often than clinical studies that are biopsy-dependent. Foroutan et al (2013) examined the ADC properties of sarcomas in mice upon treatment combination of MK1775 and gemcitabine across several days. ADC increased significantly and durably because of the combined treatment. ADC distributions demonstrated marked asymmetric patterns and a broader peak. At $24 \mathrm{~h}$ post treatment, negative skewness and kurtosis were demonstrated, whereas control groups showed positive increases. Histological measures showed a trend towards correlation of treatment response with decreased cellularity and further analysis with cleaved caspase 3 antibody staining confirmed the increased presence of apoptotic structures in tumour sections. Thus, the significant and rapid increases in ADC distribution confirm that $\mathrm{ADC}$ is a predictive marker of acute cytotoxic effects due to increased apoptosis that allow for more unrestricted diffusion.

\section{CURRENT TECHNIQUES INVESTIGATING TUMOUR HETEROGENITY WITH HISTOGRAMS}

One of the main goals of the present review is to explain the specific meaning of histogram metrics and relate them to more physiological concepts for an improved consideration of these parameters as surrogate markers of tumour heterogeneity. Some of the most used techniques for the assessment of tumour features and tumour response to treatment such as DCE-MRI, diffusionweighted MRI (DWI) as well as susceptibility-weighted MRI are reviewed in conjunction with histogram methods. For a better interpretation of histogram parameters, Table 2 summarises the overall trends emanating from recent DCE-MRI, DWI and susceptibility-weighted imaging studies.

Dynamic contrast-enhanced MRI. In 2002, Hayes et al (2002) compared for the first time, distributions of transfer constants $\left(\mathrm{K}^{\text {trans }}\right)$ in responder and non-responder patients to various treatments. Since then, the number of studies using histogrambased analysis increased drastically to address the heterogeneity of various tumour biomarkers pre- and post treatment. Indeed, the development of histogram-based analyses needed strong improvements in MRI techniques as well as modelling techniques. With increased spatial and temporal resolutions as well as improvements 
Table 2. General histogram trends in DCE-MRI, DWI-MRI, perfusion MRI and VSI-MRI studies during tumour progression and upon treatment

\begin{tabular}{|c|c|c|}
\hline Techniques & Tumour progression & Treatment \\
\hline DCE-MRI & $\begin{array}{l}\text { Skewed distributions with long tails } \\
\text { Expansion of histograms to the right } \\
\text { Peak broadening with decreased height }\end{array}$ & $\begin{array}{l}\text { Anti-angiogenic treatment, cytostatic treatment: histograms reduced to the left with } \\
\text { narrower, normal peak and increased height } \\
\text { Anti-vascular treatment: skewed histograms with increased peak height to the left }\end{array}$ \\
\hline DWI (ADC) & $\begin{array}{l}\text { Sharp histogram with high skewness and kurtosis } \\
\text { and elevated peak height }\end{array}$ & $\begin{array}{l}\text { Shift to the right side as mean ADC is increased and flatter or broader ADC histograms } \\
\text { with decreased skewness and kurtosis (King et al, 2013; Song et al, 2013) }\end{array}$ \\
\hline $\begin{array}{l}\text { Perfusion, } \\
\text { DSC-MRI (CBV) }\end{array}$ & Decreased skewness, flat CBV histograms & Increased skewness and sharp histogram (Baek et al, 2012) \\
\hline VSI-MRI & $\begin{array}{l}\text { Increased skewness to the right side of histograms, } \\
\text { increased height of peaks on the left side }\end{array}$ & $\begin{array}{l}\text { Anti-vascular treatment: decrease of 25th percentile (Burrel et al, 2012), increased } \\
\text { kurtosis }\end{array}$ \\
\hline
\end{tabular}

in signal to noise ratios and anatomical coverage, advances in alternative methods of data analysis such as histogram approaches were made possible allowing progress in the assessment of changes in tumour heterogeneity. The value of histograms was demonstrated in many early DCE-MRI studies where simple pooling of pharmacokinetic model voxel-fitted parameters and appropriate definition of thresholds revealed important spatial information in anti-angiogenic-treated tumour models and clinical studies (Chang et al, 2004; Li et al, 2005). Histogram analyses appeared to be particularly sensitive to changes in heterogeneity due to treatments. In this context, histograms of surrogate markers of permeability $\left(\mathrm{K}^{\text {trans }}\right)$ and the fractional volume of EES became a very important tool allowing the assessment of the angiogenic compositions of the whole tumour. The distributions of $\mathrm{K}^{\text {trans }}$ values allowed spatial discrimination of responses to treatment essentially between the rim and core of tumours. In solid tumours, the core is often hypoxic and/or necrotic and grows slowly compared with the highly vascularised rim. These characteristics define the aggressiveness and growth patterns of tumours and have critical implications for improved treatments. The accessibility of drugs to the tumour core is made difficult and few ways to visualise and evaluate the drug penetration are available. In many pre-clinical studies, the histographic patterns showed transformation from a broad and heterogeneous $\mathrm{K}^{\text {trans }}$ distribution to a narrow one upon anti-angiogenic treatments (Crockart et al, 2005; Jordan et al, 2005). As reported in Table 2, tumour progression appears to be linked to skewed parameter ( $\mathrm{K}^{\text {trans }}, \mathrm{Kep}$, wash in slope) distributions with long tails, expansion of histograms to the right (Increased permeability) as well as peak broadening with decreased height (Chang et al, 2004; Peng et al, 2012). All non-responders to radiotherapy in brain tumour patients demonstrated broadened histograms of the wash in slope in line with increased tumour size, whereas responders showed narrowed histogram and decreased tumour sizes (Peng et al, 2012). Histographic patterns of DCE-MRI factors after anti-angiogenic or anti-vascular treatment demonstrated skewed distributions to the left with narrower peak and increased height (Table 2). Parametric histograms were also used to characterise breast cancer heterogeneity by correlation of histograms to tumour response in a longitudinal manner during neoadjuvant chemotherapy (Chang et al, 2004). The usefulness of DCE-MRI of breast tumours for characterising tumour angiogenesis, multifocal lesions or residuals as well as allowing reliable discrimination between benign and malignant lesions as well as assessment of treatment response has been widely demonstrated. Compared with many DCE-MRI studies that use an average signaltime course or concentration-time course to assess tumour responses, Chang et al (2004) analysed the tumour composition using histograms as a function of time during neoadjuvant therapy.
A better response was associated to a larger change from heterogeneous to homogeneous distributions of amplitude with progressive increases in kurtosis and decreases in standard deviations and mean during chemotherapy. The composition of breast tumours with the highest amplitudes was the most susceptible to chemotherapy. These features may be helpful in future studies for understanding pathophysiological changes and for guiding therapeutic approaches. To date, metrics such as kurtosis, skewness and percentiles were less used in DCE-MRI clinical studies, although these parameters were predictive of radiological outcome (Chang et al, 2004) and provided improved diagnostics. DCE-MRI usually explains the kinetics of Gd-DTPA uptake within tumours using pharmacokinetic compartment modelling. Concentration-time curves are fitted to the model and estimates of permeability, tumour vessel flow, blood volume and fractional volume of EES are usually obtained (Leach et al, 2005). However, features of signal intensity-time curves can also be analysed such as gradient, overall shape, time to maximum enhancement, wash in slope and wash out that are usually more sensitive to individual variations. The initial area under the contrast-agent concentration-time curve representing a combination flow, blood volume and vessel permeability is also often used. All these model-free parameters appear to have more sensitive histogram distributions to underlying changes in the tumour environment than model-dependent parameters ( $\left.\mathrm{K}^{\text {trans }}, \mathrm{EES}, \mathrm{vp}\right)$ that have contributions from the arterial input function, the ability of the model to fit the data and remain 'indirect' surrogate markers unlike $\mathrm{ADC}$ or $\mathrm{CBV}$ (discussed below) that are more directly linked to tumour structural changes.

Diffusion MRI. During the past 10 years, DWI and diffusion tensor imaging methods have generated an increased interest for the entire MR community. In oncology, biomarkers estimated from DWI have been related to tumour aggressiveness and tumour response. Part of this interest is also because of the use of histogram approaches as they provide a quantitative methodology to analyse visible and invisible changes in tumour data in a reproducible manner (Downey et al, 2013). However, the success of diffusion techniques in turn highly contributed to the development of histogram analysis strategies. Nowadays, most studies report histogram investigations of either apparent diffusion coefficient (ADC) or fractional anisotropy in tumours (King et al; 2013; Song et al, 2013; Woo et al; 2013). Histograms are even considered to be a prerequisite in most DWI studies (Padhani et al, 2009; Ahn et al, 2012; Rosenkrantz, 2013). Histogram approaches are particularly adapted to evaluate tumour heterogeneity as they can pick up different microenvironments that may be masked by mean ADC values. ADC measures the Brownian motion of water 
molecules. In solid cancers, low ADC values correspond to restricted diffusion. In post-treatment cancers, $\mathrm{ADC}$ values are increased because of cell death and reduction of restrictive barriers but foci of necrosis may also display high ADC values. Necrotic areas are often associated to hypoxic areas resistant to radiotherapy and therefore indicating poor treatment outcome. On the other hand, increased ADC may also be due to increased interstitial osmolality and apoptosis. Anti-VEGF therapies may as well lead to reduced $\mathrm{ADC}$ values as a consequence of decreased interstitial osmolality and reduced vasogenic oedema. Histograms of ADC alone were able to identify astrocytomas from oligodendrogliomas, two glioma subtypes, by statistical comparisons of mean, mode, peak height, percentiles and skewness and by operating a classification of glioma subtypes using a discriminant analysis of each histogram parameter (Tozer et al, 2007). Again, changes in diffusivity patterns could be matched to histopathological patterns and were linked to differences in cell density. Differentiation of benign from malignant cervix tumours was possible by evaluating percentiles as well as skewness and kurtosis of ADC distributions from manually drawn ROIs around cervical tumours (Downey et al, 2013; Rosenkrantz, 2013). A significant difference was found between squamous cell carcinomas and adenocarcinomas in terms of skewness that supposedly reflects a more heterogeneous architecture of adenocarcinomas, but not with percentiles and kurtosis. The significant median ADC was able to differentiate between tumour grades. In addition, the skewness and kurtosis of ADC histograms predicted response to angiogenic therapy (bevacizumab/irinotecan) in recurrent high-grade gliomas showing that patients with increased skewness had a shorter progressionfree survival compared with patients with stable or decreased skewness (Nowosielski et al, 2011). Identification of early response in patients with newly diagnosed or recurrent ovarian cancer was possible demonstrating significantly decreased skewness and kurtosis after a third cycle of therapy (Kyriasi et al, 2011). These general histographic patterns are summarised in Table 2 for tumour progression and treatment responses and show reproducibility in a wide variety of cancers and treatments. To ensure adequate histogram distributions of ADC, efforts have to be made at the acquisition level with proper choice of $b$ values (Downey et al, 2013) and in the map segmentation to avoid contamination from CSF known to induce potential confounds. Most of the aforementioned studies provide a potential alternative to histology avoiding randomly sampled specimens and patient discomfort.

Susceptibility-weighted MRI methods: Dynamic susceptibility contrast MRI, vessel size index MRI and blood oxygen leveldependent MRI. Susceptibility-weighted MRI methods also benefited from the development of histogram-based techniques. As mentioned earlier, the chaotic development of tumour vasculature induces vascular heterogeneities in blood flow, blood volume, vessel diameters, delays and dispersion that can be investigated with DSC-MRI, VSI MRI or arterial spin labelling techniques. Cumulative histogram analysis of $\mathrm{CBV}$ with accurate choice of cutoff values not only allowed discrimination between low- and high-grade gliomas but also between grade IV and grade III gliomas with an increased diagnosis accuracy (Kim et al, 2013). CBV maps were obtained from DSC-MRI acquisitions at $3 \mathrm{~T}$ in patients with astrocytic tumours. Significant differences between low- and high-grade gliomas and between grade III and grade IV gliomas were obtained for the 99th percentile of the cumulative normalised CBV histograms, the mean and peak height.

Several recent histogram analyses of DSC-MRI and arterial spin labelling data demonstrated the usefulness and power of descriptive histogram parameters of CBV for discriminating between pseudoprogression and progression in glioblastomas (Baek et al, 2012; Choi et al, 2013; Song et al, 2013) pointing to a more recurrent use of this type of analysis in the future. However, the added value of histogram analyses can definitely be observed in studies where correlations between susceptibility-weighted MRI data and histopathological characteristics can be explored. VSI MRI and BOLD contrast MRI methodologies in rodents are particularly sensitive to structural and/or physiological factors, vessel radius and oxygenation levels for instance. Histograms of these parameters showed to be effective biomarkers of tumour heterogeneity changes (Robinson et al, 2003; Just, 2011; Burrell et al, 2012). In addition, results of histogram analysis of VSI response to anti-vascular therapy were reproducible across studies (decrease of the 25th percentile of vessel diameter) (Howe et al, 2008) and demonstrated different histographic patterns in response to treatment. Skewness, kurtosis and percentiles of the VSI and fBV distributions in different types of experimental gliomas showed significant differences between gliomas of murine origin but not between gliomas of human origin (Just, 2011) revealing the capacity of these parameters to expose microregional differences that could be of importance for providing characteristics on the tumour angiogenic phenotype. General histographic patterns for DSC-MRI and VSI-MRI are summarised in Table 2.

INTRODUCTION TO OTHER TECHNIQUES FOR EVALUATING TUMOUR HETEROGENEITY

One of the major pitfalls of histogram analysis is the poor correspondence with the spatial organisation of tumours although threshold values can be defined to correspond to parameter of interest mapping. In addition, performing statistics on distributions is not straightforward and can lead to large errors (Rose et al, 2014). Other approaches derived from histogram analysis have thus been developed. Amongst the most used are texture analysis and Réniy fractals. These techniques are often called second-order histogram analyses and take into account the joint probability distributions of pairs of pixels using parameters such as energy, entropy, correlation to evaluate the uniformity, homogeneity, internal arrangement and directionality of an image (or parameter map).

Texture analysis identifies several similar patterns in an image and groups them. In cancer MRI, it could be described as a '2Dhistogram' or co-occurrence matrix analysis (Rose et al, 2009) where the spatial information is necessary to differentiate tumour voxels with similar histograms. Rodriguez Gutierrez et al (2014) and Eliat et al (2012) were able to improve the classification of paediatric posterior fossa tumours and subtypes and differentiate between glioblastomas and malignant glioneuronal tumours, respectively, on the basis of a combination of first- and secondorder histogram features demonstrating improved specificity and sensitivity for differentiating tumours. These studies emphasised the usefulness of texture-based classification techniques in identifying tumour subtypes. In particular, optimisation of texture analysis may help discrimination between genetic tumour subtypes (Rodriguez Gutierrez et al, 2014 and references therein) in agreement with improvements of patient comfort concepts encouraging the development of noninvasive cancer classification.

Fractal dimensions (Rose et al, 2009) have also been used to add knowledge of spatial arrangement in tumours to usual DCE-MRI parameter distributions allowing to describe 'heterogeneity biomarkers'. Fractal dimensions are model-based tools for texture analysis describing objects that have a high degree of irregularity. Rose et al (2009) used fractal dimensions as well as geometrical measurements to quantify random variations in DCE-MRI parameter maps of low- and high-grade gliomas. Their methodology comparing spatial heterogeneity statistics (information dimension, extruded surface area, etc.) for $\mathrm{K}^{\text {trans }}$ and EES parameters showed increased statistical difference $(P<0.00005)$ for discriminating low-grade gliomas from high-grade gliomas compared with 
more conventional statistics (median and standard deviation) $(P<0.05)$.

Finally Rose et al (2014) also addressed the fundamental problem of statistical significance of parametric tumour maps using indexed distribution analysis of bevacizumab DCE-MRI data. Compared with conventional and histogram analyses, indexed distribution analysis was sensitive to heterogeneous changes and spatial extent of bevacizumab treatment, even allowing inference about treatment-induced decrease in $\mathrm{K}^{\text {trans }}$ independence on baseline values.

However, all these new methodologies require further validation and are still based on previous evaluations of parameter distributions. The validation of these biomarkers will require the development of simple standardised methodologies across centres and the creation of databanks including cancer types and subtypes, treatments and parameters. One of the main pitfalls of histogram and histogram-derived approaches concerns the retrospective nature of most of the studies where histopathological features of tumours are known and can bias the true usefulness of these novel approaches. Another main limitation appears to be the low number of patients bearing a specific type of cancer. Repeated and reproducible trends need to be extracted from histogram factors (such as kurtosis and skewness changes, for example) to become key points for appropriate therapeutic decisions (Table 2).

\section{CONCLUSION AND FUTURE CHALLENGES}

For the past 15-20 years, advances in cancer characterisation and assessment of response to treatment have been correlated to advances in cancer imaging techniques. A vast majority of studies have focused on the assessment of surrogate biomarkers of various parameters (permeability $\left(\mathrm{K}^{\text {trans }}\right)$, blood flow, diffusivity (ADC), vessel diameter (VSI), etc.) allowing either grading of tumours, discrimination between tumour types, benign or malignant lesions or assessment of early response to treatment following antiangiogenic/anti-vascular or on the contrary progression of cancer, metastatic outcome or patient outcome. Lately, progress in genetic, cellular and molecular technologies allowed addressing tumour heterogeneity in a more comprehensive manner with deepened knowledge of genetic mutations of tumours and their subtypes. These studies have generated a lot of interest as the increased understanding of tumour heterogeneous environment is directly implicated in the development of novel targeted therapies, their combination and their clinical therapeutic schemes as well as the interpretation of tumour resistance to therapy. In imaging-based oncology research, although parametric voxel-by-voxel maps have been used for a long time allowing visualisation of heterogeneity, parameters have often been summarised into average or median scalars that described only discrete aspects of the distributions of parameters across tumours and were not even reflecting the underlying biology. Descriptive first-order histogram approaches remain simple and accessible to many investigators. Recently, a wide range of clinical and pre-clinical studies have demonstrated the potential of these analyses for grading, differentiating, assessing progression and tumour responses. Increased specificity, sensitivity and accuracy of histogram parameters have often been shown compared with conventional or histopathological analyses. Metrics such as kurtosis, skewness, percentiles and their changes demonstrated to be strong and reliable quantitative surrogate markers of tumour heterogeneity with a more direct correlation to the underlying structural, physiological, molecular as well as metabolic changes occurring upon tumour progression with and without therapy. Texture analysis and fractals further improve quantitative histogram approaches by introducing the spatial dimension allowing better classification or grading. These later methodologies are computer-demanding and need further validations although they should provide high-quality diagnosis of tumour subtypes. Critical challenges for the future involve validation and standardisation of quantitative histogram approaches in large patient populations as a reliable tool to characterise and classify tumour heterogeneity non-invasively.

ACKNOWLEDGEMENTS

I would like to thank the reviewers for highly contributing to the improvements of this manuscript. Dr Nathalie Just was supported by the Centre d'Imagerie BioMédicale (CIBM) of the Ecole Polytechnique Fédérale de Lausanne (EPFL) and the Leenards and Jeantet foundations.

\section{REFERENCES}

Ahn SJ, Choi SH, Kim YJ, Kim KG, Sohn CH, Han MH, Chang KH, Min HS (2012) Histogram analysis of apparent diffusion coefficient map of standard and high B-value diffusion MR imaging in head and neck squamous cell carcinoma: a correlation study with histological grade. Acad Radiol 19: 1233-1240.

Baek HJ, Kim HS, Kim N, Choi YJ, Kim YJ (2012) Percent change of perfusion skewness and kurtosis: a potential imaging biomarker for early treatment response in patients with newly diagnosed glioblastomas. Radiology 264 : 834-843.

Burrell JS, Bradley Robert S, Walker-Samuel Simon, Jamin Yann, Baker Lauren CJ, Boult Jessica KR, Withers Philip J, Halliday Jane, Waterton John C, Robinson Simon P (2012) MRI measurements of vessel calibre in tumor xenografts: Comparison with vascular corrosion casting. Microvasc Res 84: 323-329.

Carter JS, Koopmeiners JS, Kuehn-Hajder JE, Metzger GJ, Lakkadi N, Downs Jr LS, Bolan PJ (2013) Quantitative multiparametric MRI of ovarian cancer. J Magn Reson Imaging 38: 1501-1509.

Chandarana H, Rosenkrantz AB, Mussi TC, Kim S, Ahmad AA, Raj SD, McMenamy J, Melamed J, Babb JS, Kiefer B, Kiraly AP (2012) Histogram analysis of whole-lesion enhancement in differentiating clear cell from papillary subtype of renal cell cancer. Radiology 265: 790-798.

Chang YC, Huang CS, Liu YJ, Chen JH, Lu YS, Tseng WY (2004) Angiogenic response of locally advanced breast cancer to neoadjuvant chemotherapy evaluated with parametric histogram from dynamic contrast-enhanced MRI. Phys Med Biol 49: 3593-3602.

Choi YJ, Kim HS, Jahng GH, Kim SJ, Suh DC (2013) Pseudoprogression in patients with glioblastoma: added value of arterial spin labeling to dynamic susceptibility contrast perfusion MR imaging. Acta Radiol 54(4): $448-454$.

Crokart N, Radermacher K, Jordan BF, Baudelet C, Cron GO, Grégoire V, Beghein N, Bouzin C, Feron O, Gallez B (2005) Tumor radiosensitization by anti-inflammatory drugs: evidence for a new mechanism involving the oxygen effect. Cancer Res 65: 7911-7916.

De Sousa EMF, Vermeulen L, Fessler E, Medema JP (2013) Cancer heterogeneity-a multifaceted view. EMBO Rep 14(8): 686-695.

Downey K, Riches SF, Morgan VA, Giles SL, Attygalle AD, Ind TE, Barton DP, Shepherd JH, deSouza NM (2013) Relationship between imaging biomarkers of stage I cervical cancer and poor-prognosis histologic features: quantitative histogram analysis of diffusion-weighted MR images. AJR Am J Roentgenol 200: 314-320.

Eliat PA, Olivié D, Saïkali S, Carsin B, Saint-Jalmes H, de Certaines JD (2012) Can dynamic contrast-enhanced magnetic resonance imaging combined with texture analysis differentiate malignant glioneuronal tumors from other glioblastoma? Neurol Res Int 2012: 195176.

Foroutan P, Kreahling JM, Morse DL, Grove O, Lloyd MC, Reed D, Raghavan M, Altiok S, Martinez GV, Gillies RJ (2013) Diffusion MRI and novel texture analysis in osteosarcoma xenotransplants predicts response to anti-checkpoint therapy. PLoS One 8(12): e82875.

Friedman SN, Bambrough PJ, Kotsarini C, Khandanpour N, Hoggard N (2012) Semi-automated and automated glioma grading using dynamic susceptibility-weighted contrast-enhanced perfusion MRI relative cerebral blood volume measurements. Br J Radiol 85: e1204-e1211. 
Hayes C, Padhani AR, Leach MO (2002) Assessing changes in tumor vascular function using dynamic contrast-enhanced magnetic resonance imaging. NMR Biomed 15: 154-163.

Howe FA, McPhail LD, Griffiths JR, McIntyre DJ, Robinson SP (2008) Vessel size index magnetic resonance imaging to monitor the effect of antivascular treatment in a rodent tumor model. Int J Radiat Oncol Biol Phys 71: 1470-1476.

Johansen R, Jensen L, Rydland J, Goa PE, Kvistad KA, Bathen TF, Axelson DE, Lundgren S, Gribbestad IS (2009) Predicting survival and early clinical response to primary chemotherapy for patients with locally advanced breast cancer using DCE-MRI. J Magn Reson Imaging 29: 1300-1307.

Jordan BF, Runquist M, Raghunand N, Gillies RJ, Tate WR, Powis G, Baker AF (2005) The thioredoxin-1 inhibitor 1-methylpropyl 2-imidazolyl disulfide (PX-12) decreases vascular permeability in tumor xenografts monitored by dynamic contrast enhanced magnetic resonance imaging. Clin Cancer Res 11: 529-536.

Just N (2011) Histogram analysis of the microvasculature of intracerebral human and murine glioma xenografts. Magn Reson Med 65: 778-789.

Kim H, Choi SH, Kim JH, Ryoo I, Kim SC, Yeom JA, Shin H, Jung SC, Lee AL, Yun TJ, Park CK, Sohn CH, Park SH (2013) Gliomas: application of cumulative histogram analysis of normalized cerebral blood volume on 3T MRI to tumor grading. PLoS One 21: e63462.

King AD, Chow KK, Yu KH, Mo FK, Yeung DK, Yuan J, Bhatia KS, Vlantis AC, Ahuja AT (2013) Head and neck squamous cell carcinoma: diagnostic performance of diffusion-weighted MR imaging for the prediction of treatment response. Radiology 266: 531-538.

Kyriazi S, Collins DJ, Messiou C, Pennert K, Davidson RL, Giles SL, Kaye SB, Desouza NM (2011) Metastatic ovarian and primary peritoneal cancer: assessing chemotherapy response with diffusion-weighted MR imagingvalue of histogram analysis of apparent diffusion coefficients. Radiology 261: 182-192.

Leach MO, Brindle KM, Evelhoch JL, Griffiths JR, Horsman MR, Jackson A, Jayson GC, Judson IR, Knopp MV, Maxwell RJ, McIntyre D, Padhani AR, Price P, Rathbone R, Rustin GJ, Tofts PS, Tozer GM, Vennart W, Waterton JC, Williams SR, Workman P. Pharmacodynamic/ Pharmacokinetic Technologies Advisory Committee, Drug Development Office, Cancer Research UK (2005) The assessment of antiangiogenic and antivascular therapies in early-stage clinical trials using magnetic resonance imaging: issues and recommendations. Br J Cancer 92(9): $1599-1610$

Li KL, Wilmes LJ, Henry RG, Pallavicini MG, Park JW, Hu-Lowe DD, McShane TM, Shalinsky DR, Fu YJ, Brasch RC, Hylton NM (2005) Heterogeneity in the angiogenic response of a BT474 human breast cancer to a novel vascular endothelial growth factor-receptor tyrosine kinase inhibitor: assessment by voxel analysis of dynamic contrast-enhanced MRI. J Magn Reson Imaging 22: 511-519.

Mignion L, Dutta P, Martinez GV, Foroutan P, Gillies RJ, Jordan BF (2014) Monitoring chemotherapeutic response by hyperpolarized 13C-fumarate MRS and diffusion MRI. Cancer Res 74(3): 686-694.

Nowosielski M, Recheis W, Goebel G, Güler O, Tinkhauser G, Kostron H, Schocke M, Gotwald T, Stockhammer G, Hutterer M (2011) ADC histograms predict response to anti-angiogenic therapy in patients with recurrent high-grade glioma. Neuroradiology 53: 291-302.

Padhani AR, Liu G, Koh DM, Chenevert TL, Thoeny HC, Takahara T, Dzik-Jurasz A, Ross BD, Van Cauteren M, Collins D, Hammoud DA, Rustin GJ, Taouli B, Choyke PL (2009) Diffusion-weighted magnetic resonance imaging as a cancer biomarker: consensus and recommendations. Neoplasia 11: 102-125.
Padhani AR (2002) Dynamic contrast-enhanced MRI in clinical oncology: current status and future directions. J Magn Reson Imaging 16: 407-422.

Peng SL, Chen CF, Liu HL, Lui CC, Huang YJ, Lee TH, Chang CC, Wang FN (2012) Analysis of parametric histogram from dynamic contrast-enhanced MRI: application in evaluating brain tumor response to radiotherapy. NMR Biomed 26: 443-450.

Robinson SP, Rijken PF, Howe FA, McSheehy PM, van der Sanden BP, Heerschap A, Stubbs M, van der Kogel AJ, Griffiths JR (2003) Tumor vascular architecture and function evaluated by non-invasive susceptibility MRI methods and immunohistochemistry. J Magn Reson Imaging 17: $445-454$.

Rodriguez Gutierrez D, Awwad A, Meijer L, Manita M, Jaspan T, Dineen RA, Grundy RG, Auer DP (2014) Metrics and textural features of mri diffusion to improve classification of pediatric posterior fossa tumors. AJNR Am J Neuroradiol 35(5): 1009-1015.

Rose CJ, Mills SJ, O'Connor JP, Buonaccorsi GA, Roberts C, Watson Y, Cheung S, Zhao S, Whitcher B, Jackson A, Parker GJ (2009) Quantifying spatial heterogeneity in dynamic contrast-enhanced MRI parameter maps. Magn Reson Med 62(2): 488-499.

Rose CJ, O'Connor JP, Cootes TF, Taylor CJ, Jayson GC, Parker GJ, Waterton JC (2014) Indexed distribution analysis for improved significance testing of spatially heterogeneous parameter maps: Application to dynamic contrast-enhanced MRI biomarkers. Magn Reson Med 71(3): 1299-1311.

Rosenkrantz AB (2013) Histogram-based apparent diffusion coefficient analysis: an emerging tool for cervical cancer characterization? AJR Am J Roentgenol 200: 311-313.

Shukla-Dave A, Lee NY, Jansen JF, Thaler HT, Stambuk HE, Fury MG, Patel SG, Moreira AL, Sherman E, Karimi S, Wang Y, Kraus D, Shah JP, Pfister DG, Koutcher JA (2012) Dynamic contrast-enhanced magnetic resonance imaging as a predictor of outcome in head-and-neck squamous cell carcinoma patients with nodal metastases. Int J Radiat Oncol Biol Phys 82: 1837-1844.

Song YS, Choi SH, Park CK, Yi KS, Lee WJ, Yun TJ, Kim TM, Lee SH, Kim JH, Sohn CH, Park SH, Kim IH, Jahng GH, Chang KH (2013) True progression versus pseudoprogression in the treatment of glioblastomas: a comparison study of normalized cerebral blood volume and apparent diffusion coefficient by histogram analysis. Korean J Radiol 14(4): 662-672.

Tozer DJ, Jäger HR, Danchaivijitr N, Benton CE, Tofts PS, Rees JH, Waldman AD (2007) Apparent diffusion coefficient histograms may predict low-grade glioma subtype. NMR Biomed 20: 49-57.

Woo S, Cho JY, Kim SY, Kim SH (2013) Histogram analysis of apparent diffusion coefficient map of diffusion-weighted MRI in endometrial cancer: a preliminary correlation study with histological grade. Acta Radiol; e-pub ahead of print 6 December 2013; pii0284185113514967.

Yuh WT, Mayr NA, Jarjoura D, Wu D, Grecula JC, Lo SS, Edwards SM, Magnotta VA, Sammet S, Zhang H, Montebello JF, Fowler J, Knopp MV, Wang JZ (2009) Predicting control of primary tumor and survival by DCE MRI during early therapy in cervical cancer. Invest Radiol 44: 343-350.

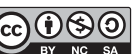

This work is licensed under the Creative Commons Attribution-NonCommercial-Share Alike 3.0 Unported License. To view a copy of this license, visit http://creativecommons. org/licenses/by-nc-sa/3.0/ 